\title{
Modeling multiturn stripping injection and foil heating for high intensity proton drivers
}

\author{
A. I. Drozhdin, ${ }^{*}$ I. L. Rakhno, ${ }^{\dagger}$ S. I. Striganov, ${ }^{*}$ and L. G. Vorobiev ${ }^{\S}$ \\ Fermi National Accelerator Laboratory, Batavia, Illinois 60510, USA
}

(Received 2 November 2011; published 20 January 2012)

\begin{abstract}
$\mathrm{H}^{-}$stripping injection into the Fermilab recycler ring, combined with a beam phase painting technique, has been considered. The multiparticle three-dimensional beam dynamics with space charge has been studied numerically, using STRUCT and ORBIT codes, for different painting scenarios. In order to achieve a uniform (quasi-KV) phase-space distribution and to reduce the foil heating, the following parameters were investigated: the number of turns, strengths and temporal forms of kicker magnets, and foil geometry. Performance of the stripping foil is a crucial parameter of the whole injection scheme, so that the latter has been designed to minimize the hit number on the foil. The temperature regime has been evaluated both semianalytically and numerically using Monte Carlo codes MARS and MCNPX, with radiation cooling and transport of $\delta$ electrons taken into account. That all results agreed well proves the consistency of the models. It has been shown that the stripping foil can survive during injection with the parameters chosen for Project X at Fermilab.
\end{abstract}

DOI: 10.1103/PhysRevSTAB.15.011002

PACS numbers: 29.27.Ac, 41.85.Ar

\section{INTRODUCTION}

Painting injection with a stripping foil is a well established technique implemented at KEK and other accelerator laboratories. The stripping, when $\mathrm{H}^{-}$charge exchange occurs, allows one to overcome the limitations imposed by the Liouville's theorem and the proper strengths of painting magnets help to form a desired phase-space beam distribution. For high-current accelerators, the most preferable phase density is a quasi-KV distribution in the transverse $\left(x, x^{\prime}, y, y^{\prime}\right)$ plane which is superior compared to other nonuniform distributions due to minimal tune shift. Longitudinal painting, resulting in a uniform shape of the density in $(\Delta \phi, \Delta E)$ phase space, is also preferable.

On the one hand, it is easier to build a distribution for many turns, but on the other hand, the foil heating suggests having a faster injection with fewer turns, when the beam phase profiles may still be inappropriate. Therefore, the injection scheme has to represent a compromise between the resulting beam phase distribution, which is important for further dynamics, and foil sustainability, defined by tolerable heating of foil material. Other foil issues, such as secondary electrons and neutral $\mathrm{H}^{0}$ species produced in

\footnotetext{
*drozhdin@fnal.gov

†rakhno@fnal.gov

striganov@fnal.gov

§lgv@fnal.gov
}

Published by the American Physical Society under the terms of the Creative Commons Attribution 3.0 License. Further distribution of this work must maintain attribution to the author(s) and the published article's title, journal citation, and DOI. the foil, may seriously complicate the injection resulting in additional losses.

Several approaches have been used to simulate the $\mathrm{H}^{-}$ stripping on the foil due to multiple Coulomb scattering, nuclear interactions, etc. The numerical simulations agreed well with a semianalytical model, giving confidence that for the chosen injection scheme parameters the stripping foil will survive.

\section{PAINTING INJECTION}

In this section we discuss the basics of painting instrumentation and its numerical aspects in application to multiturn injection into the recycler ring (RR) (see Refs. [1,2]).

For different painting cycle durations we evaluated and compared the resulting emittances and the number of hits of the stripping foil. This information will be utilized for the detailed modeling of stripping foil performance in Secs. III and IV.

\section{A. General layout and basics of the painting technique}

A layout of the injection scheme of $\mathrm{H}^{-}$ions from the $8 \mathrm{GeV}$ linac into the Fermilab recycler ring is illustrated in Figs. 1 and 2. One can see in Fig. 1 how $\mathrm{H}^{-}$beam hits the stripping foil and is then merging with the closed orbit of the recycler. The permanent bump magnets, denoted as $\mathrm{HBC} 1$ through $\mathrm{HBC} 4$, provide the offset of the closed orbit (all bumps have a constant strength) to circumvent the body size of the RR dipole magnet (not shown in Fig. 1) installed upstream of the injection point. The bump magnets $\mathrm{HBC} 3$ and $\mathrm{HBC} 4$ are also designed to send a small amount of $\mathrm{H}^{ \pm}$and $\mathrm{H}^{0}$ after the stripping into absorbers. 


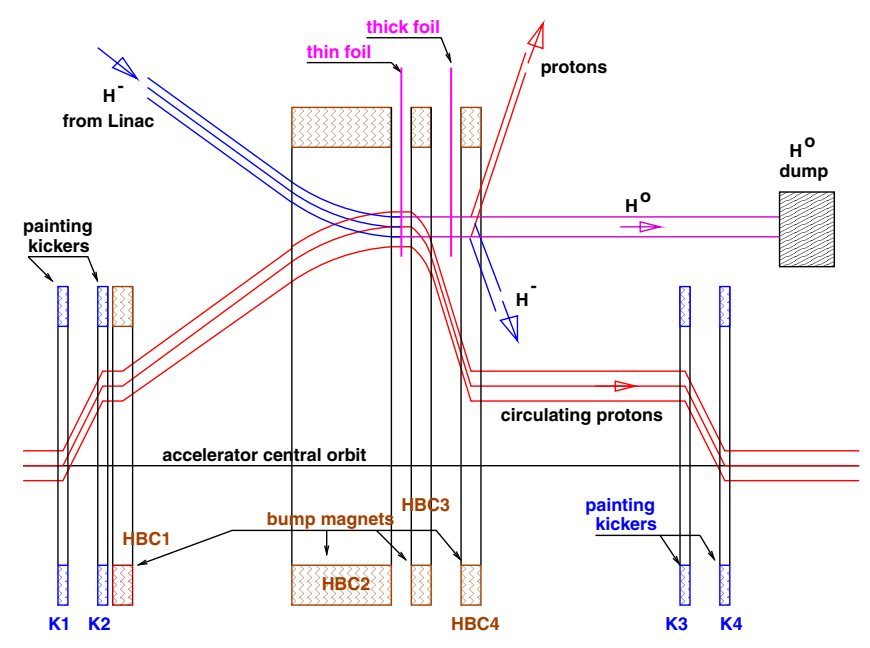

FIG. 1. Painting injection scheme.

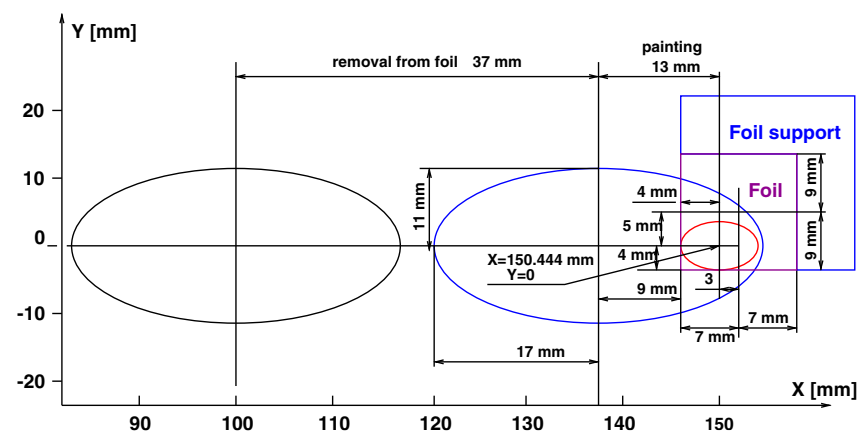

FIG. 2. Injected and circulating beam locations at the $3-\mu \mathrm{m}$ foil during painting. The transverse dimensions of the foil are $14 \mathrm{~mm} \times 18 \mathrm{~mm}$.

Only fast kicker magnets, denoted as K1 through K4, play an active role in painting. The magnets arrange a timevarying beam steering on the stripping foil, which is called the "painting." The painting procedure assumes a stripping, combined with the injection with different coordinates $(x, y)$ and under different angles $\left(x^{\prime}, y^{\prime}\right)$, to build a designated transverse phase-space distribution. Stripping plays a key role in that procedure. It allows one to increase turn after turn the intensity of the circulating beam without a significant growth of the transverse phase volume, and also allows building a phase distribution with desired parameters. All this happens because the stripping allows one to inject the particles in the same cell of the $\left(x, x^{\prime}, y, y^{\prime}\right)$ phase space repeatedly.

The $3-\mu \mathrm{m}\left(600 \mu \mathrm{g} / \mathrm{cm}^{2}\right)$ thick graphite stripping foil has the shape of a so-called "corner foil," where two edges of the square foil are supported and the other two edges are free.

\section{B. Simulations without space charge}

The results presented in this section were obtained with the computer code STRUCT [3]. The normalized emittance of injected beam at $95 \%$ level is equal to $2.5 \mathrm{~mm}$ mrad. The circulating beam emittance after painting is $\varepsilon_{3 \sigma}=$ $37.5 \mathrm{~mm}$ mrad. In the chosen scheme, the painting lasts for 582 turns and after the painting the circulating beam is removed from the foil over seven turns. The horizontal bump amplitude at the foil is: $50 \mathrm{~mm}=13 \mathrm{~mm}$ (painting) $+37 \mathrm{~mm}$ (removing from the foil) (Fig. 2).

The proton orbit is moved in the horizontal plane at the beginning of injection by $150 \mathrm{~mm}$ to the stripping foil to accept the first portion of protons generated by $\mathrm{H}^{-}$in the foil (Fig. 1). The horizontal and vertical kickers are used to produce orbit displacement during painting. A gradual reduction of horizontal kicker strength permits painting the injected beam across the accelerator aperture with the required emittance. The vertical kicker magnets located in the injection line (not shown) provide injected beam angle, sweeping during injection from the maximum at the beginning down to zero at the end of the painting process (Fig. 3). The vertical angle variation is $0.26 \mathrm{mrad}$. Painting starts from the central region of phase space in the horizontal plane and from the border of it in the vertical plane, and goes first to the border of the beam in the horizontal plane and then to the center in the vertical plane. This produces a so-called "uncorrelated painting" with elliptical beam cross section, thereby eliminating particles that have maximum amplitudes in both planes simultaneously. Painting injection scenarios studied for accumulation of $1.47 \times 10^{14} \mathrm{ppp}$ in the recycler ring are presented in Table II. The short injector linac pulse length (1-5 ms) requires six injections to accumulate the required intensity in the recycler ring. The dependence of kickermagnets' strength on time (Fig. 4) is chosen to get a uniform distribution [4] of the beam after painting both in horizontal and vertical planes (Fig. 5). Particle hit distributions in the foil at the 1st, 4th, and 6th cycle, and during all six cycles of the 582-turn injection are shown in Fig. 6.

A low field magnet $\mathrm{HBC} 2$, located upstream of the foil (Table I), is used to separate the proton and $\mathrm{H}^{-}$beam at the

TABLE I. Injection magnet parameters.

\begin{tabular}{lcccc}
\hline \hline Name & $L[m]$ & \multirow{2}{*}{$B[k G]$} & \multicolumn{2}{c}{ Half aperture $[\mathrm{mm}]$} \\
& & $X$ & $Y$ \\
\hline \multicolumn{2}{l}{ Kicker magnets } & & & \\
K1 & 0.5 & -10.981 & 76.2 & 25.4 \\
K2 & 0.5 & 10.981 & 76.2 & 25.4 \\
K3 & 0.5 & 10.980 & 76.2 & 25.4 \\
K4 & 0.5 & -10.980 & 76.2 & 25.4 \\
Bump magnets & & & \\
HBC1 & 0.7 & -4.26010 & 76.2 & 25.4 \\
HBC2 & 6.0 & 0.54643 & 200.0 & 38.1 \\
HBC3 & 1.0 & 12.25700 & 200.0 & 38.1 \\
HBC4 & 1.0 & -12.55400 & 200.0 & 38.1 \\
\hline \hline
\end{tabular}


TABLE II. Painting injection scenarios studied for accumulation of $1.47 \times 10^{14}$ protons per pulse (ppp) in the recycler ring.

\begin{tabular}{|c|c|c|c|c|}
\hline Scenario & $A$ & $B$ & $C$ & $D$ \\
\hline Linac repetition rate $[\mathrm{Hz}]$ & 10 & 10 & 10 & 0.71 \\
\hline Painting cycle duration [ms] & 1.08 & 2.16 & 4.28 & 25.72 \\
\hline Number of RR turns per painting cycle & 97 & 194 & 385 & 2310 \\
\hline Number of painting cycles per injection & 6 & 6 & 6 & 1 \\
\hline Interval between painting cycles [ms] & 98.92 & 97.84 & 95.72 & \\
\hline Total injection time $[\mathrm{ms}]$ & 501.08 & 502.16 & 504.28 & 25.72 \\
\hline Total number of turns on foil during injection & 582 & 1164 & 2310 & 2310 \\
\hline Injection intensity per RR turn & $2.52 \times 10^{11}$ & $1.26 \times 10^{11}$ & $6.30 \times 10^{10}$ & $6.30 \times 10^{10}$ \\
\hline Average number of each proton interaction with foil during injection & 32.6 & 60.0 & 118.0 & 118.0 \\
\hline
\end{tabular}

quadrupole preceding the injection region. This magnetic field of $\sim 500 \mathrm{G}$ is chosen to prevent field stripping of $\mathrm{H}^{-}$ before interaction with the foil. The second thick foil, located downstream of the third horizontal magnet
$\mathrm{HBC} 3$, provides stripping to protons of the final $\mathrm{H}^{0}$ atoms, and the fourth horizontal magnet $\mathrm{HBC} 4$ located behind the stripping foil is used for removal of these protons to the beam absorber.
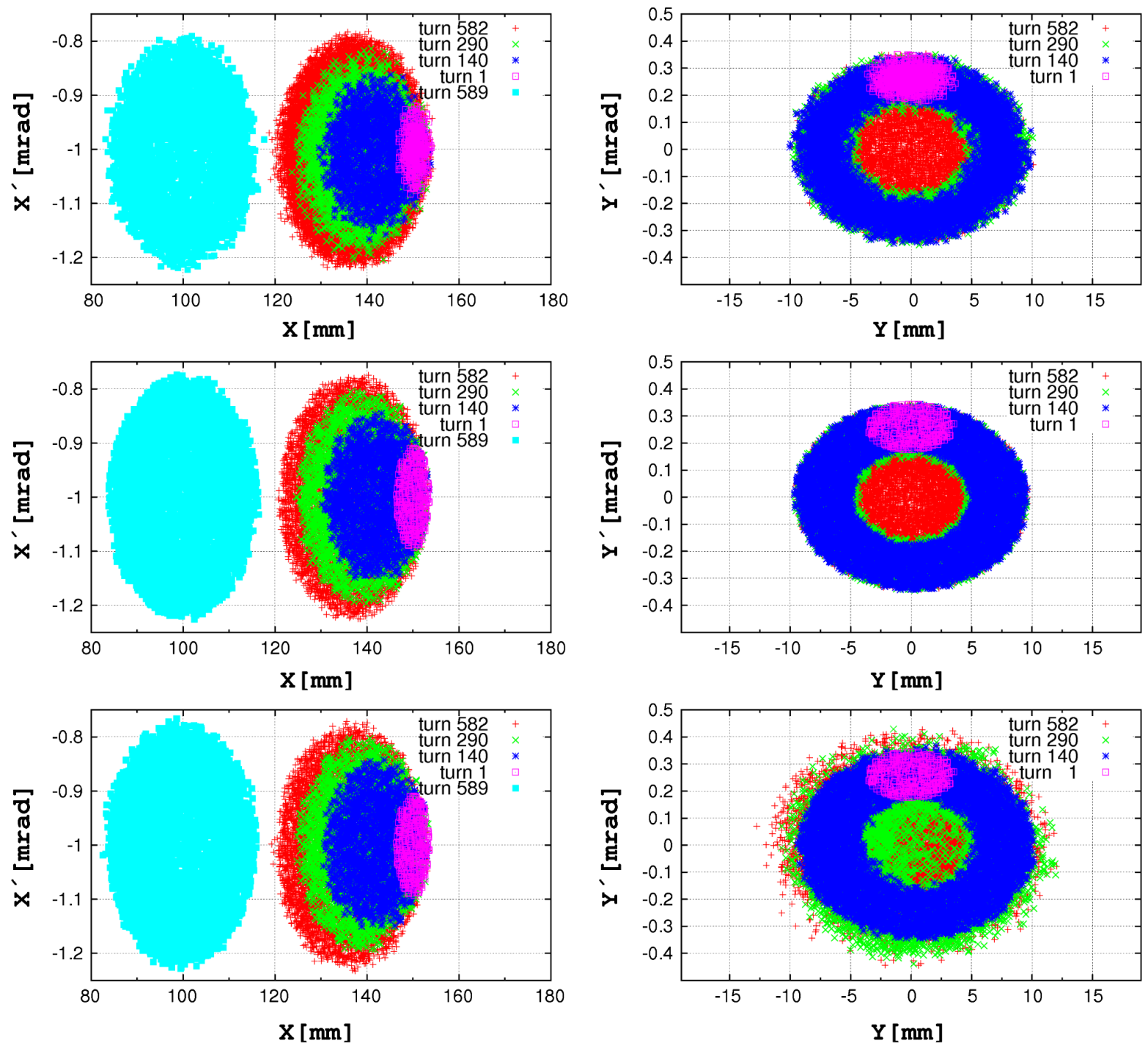

FIG. 3. Horizontal $\left(x, x^{\prime}\right)$ and vertical $\left(y, y^{\prime}\right)$ phase portraits of the circulating beam at the first, 140th, 290th, 582nd, and 589th turns of injection, simulated by STRUCT (top), ORBIT with zero current (middle), and ORBIT with full current (bottom). 


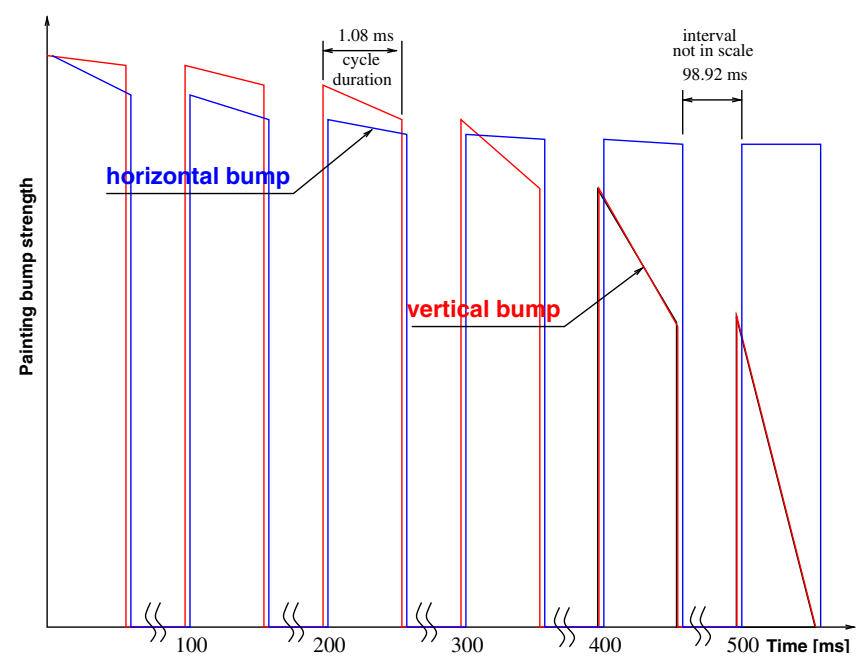

FIG. 4. Horizontal and vertical painting bump functions during injection.

\section{Simulations with space charge effects}

We benchmarked the results of the STRUCT code from Sec. II B using the code ORBIT [5] which simulates three-dimensional beam dynamics in accelerator and storage rings. The ORBIT code imports the accelerator lattice in MAD format [6] and initial phase portrait $\left(x, x^{\prime}, y, y^{\prime}, \Delta \phi, \Delta E\right)$ of the particle distribution, and then tracks macroparticles through the lattice, taking into account acceleration in $\mathrm{rf}$ cavities, foil stripping phenomena, and longitudinal and transverse space charge forces.

First, we omitted the space charge effects and made a comparison with Sec. II B. In Fig. 3 (middle plots) the transverse phase-space coordinates are plotted for zero current mode, which are in a good agreement with results obtained with STRUCT.

The next step was to take into account space charge. The total number of protons circulating in the recycler after injection is $1.5 \times 10^{14}$; that corresponds to the total beam current accumulated after injection in the recycler ring equal to $2.1 \mathrm{~A}$. When we switch on the full current the space charge effects on beam dynamics are expected to be important. In our simulations we were tracking $\sim 2 \times 10^{5}$ macroparticles (after 582 turns, with 360 macroparticles injected on each turn) and were using a spatial grid of $128 \times 128 \times 64$ in $x, y$, and $z$ dimension, respectively. In Fig. 3 (bottom plots), one can see transverse phase portraits for the same turn numbers, when the beam current is $2.1 \mathrm{~A}$. Let us compare middle and bottom plots in Fig. 3. Interestingly enough, it looks like the phase portraits in the horizontal phase space $\left(x, x^{\prime}\right)$ did not change much. In the vertical space $\left(y, y^{\prime}\right)$ the situation looks different: the phase pictures with zero and full current differ noticeably. This observation does not mean, though, that space charge manifests itself more strongly in the $\left(y, y^{\prime}\right)$ plane and the horizontal phase coordinates $\left(x, x^{\prime}\right)$ are insensitive to the beam current.

In fact, this asymmetry depends on the way the horizontal and vertical painting are realized. In the $\left(x, x^{\prime}\right)$ plane injection occurs from the center (i.e., for the small amplitude particles) towards outside, to the larger amplitude particles. In the vertical phase space $\left(y, y^{\prime}\right)$ the painting starts from the larger amplitude particles towards the center, to the smaller amplitude particles. Therefore, the peripheral particles $\left(y, y^{\prime}\right)$ experience growing space charge effects during the whole injection cycle. Of course, in the horizontal plane the particles are also affected by the Coulomb force. However, the peripheral ones $\left(x, x^{\prime}\right)$ are experiencing the space charge effect over fewer turns and do not have enough time to demonstrate any significant difference in comparison with the zero beam current calculations. In the meantime, after more turns the dilution of marginal coordinates $\left(x, x^{\prime}\right)$ due to high current becomes quite noticeable.

One can see also the longitudinal beam dynamics. For zero and maximal beam current the distributions in Fig. 7 are very much alike. According to the multiturn injection scenario, a $325 \mathrm{MHz}$ linac beam is injected into RR over 582 turns, hitting the foil repeatedly. The $52.8 \mathrm{MHz}$
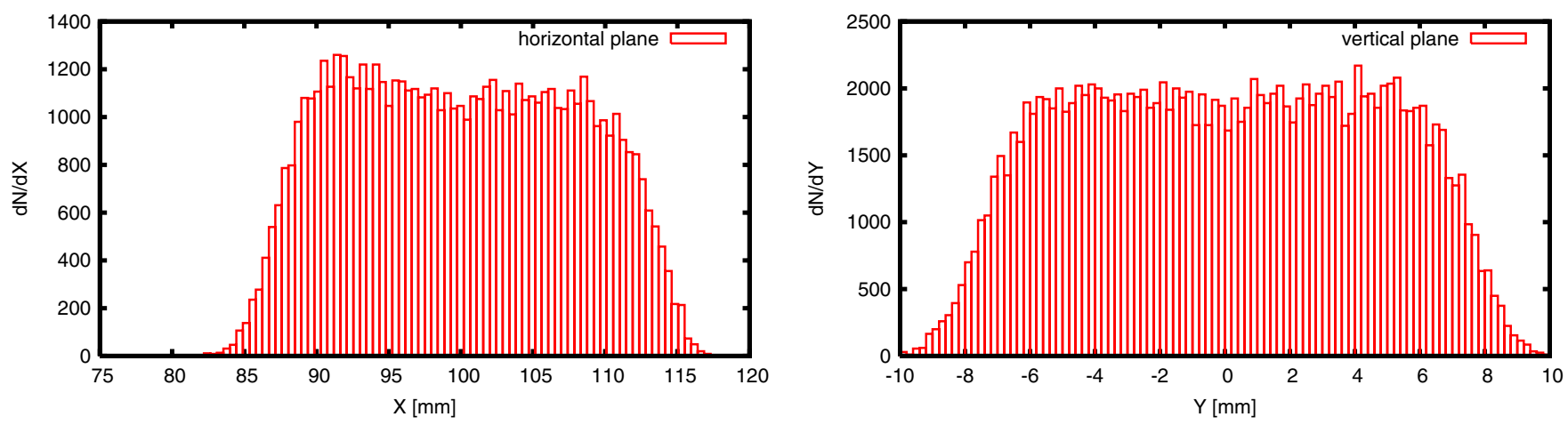

FIG. 5. Horizontal (left) and vertical (right) particle distributions after painting. 

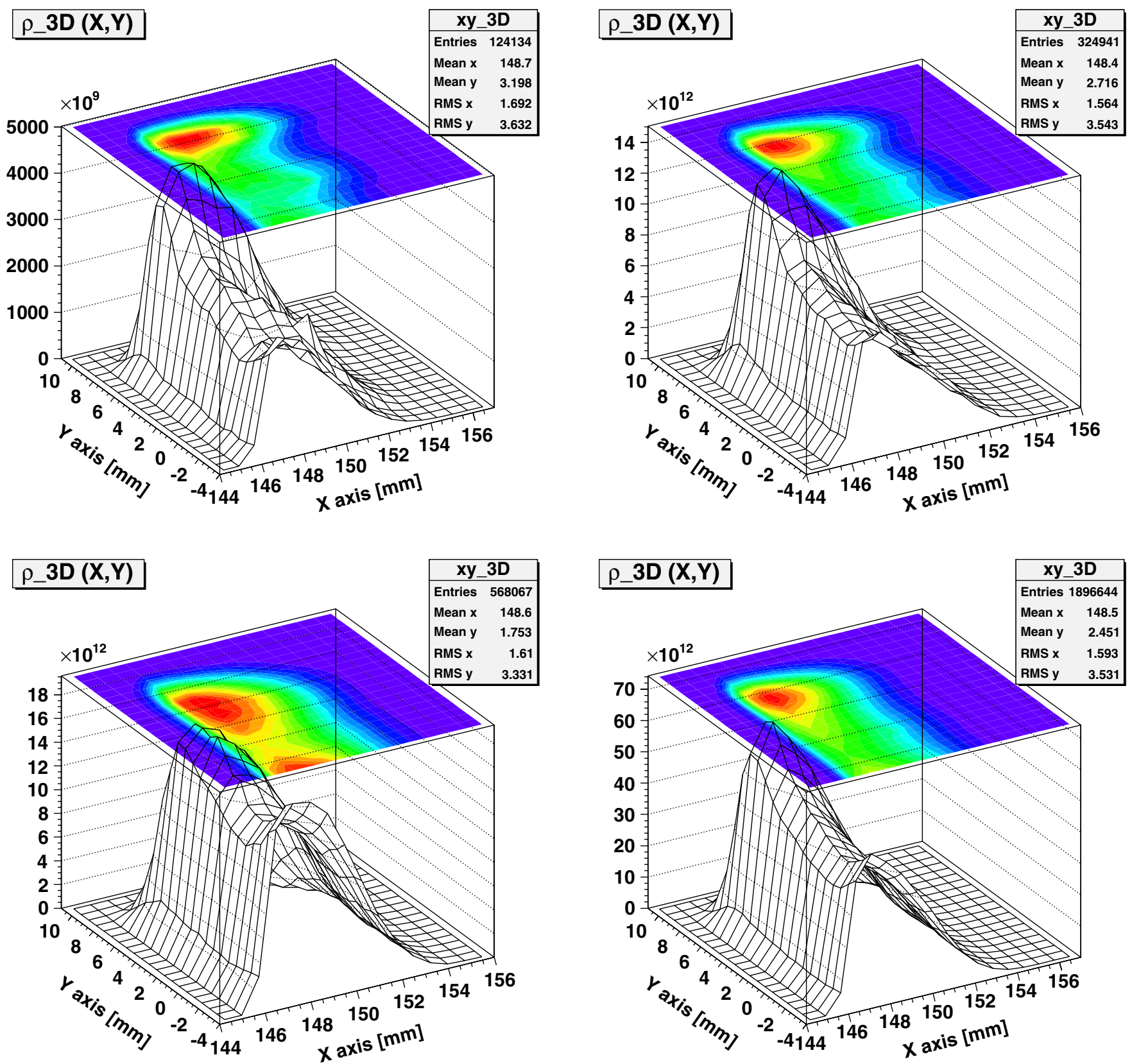

FIG. 6. Hit distributions in the foil at the 1st (top, left), 6th (top, right), 4th (bottom, left), and during all the six (bottom, right) cycles of the 582-turn injection. In the simulations, the particle hit number on the foil during the 1 st, 4 th, and 6 th cycles is 62067,162470 , and 284034 , respectively. The total hit number during the injection is 948322 . Average number of interactions with the foil for each injected particle is 33 . Hit density at the maximum of the distribution is $1.31 \times 10^{14}$ proton $/ \mathrm{mm}^{2}$ at $2.52 \times 10^{11}$ particles injected at every turn.

rf cavities in the recycler work with two harmonic voltages of $400 \mathrm{kV}$ and $-200 \mathrm{kV}$. Since the ratio of linac vs recycler rfs is not an integer, $325 / 52.8=6.15$, the fractional part, 0.15 , results in a slipping of linac microbunches along the rf phase, as demonstrated in Fig. 7. Although the recycler separatrix (with phases $[-\pi, \pi]$ ) is able to accommodate six microbunches, in practice there are only four with range $[-2.05,2.05]$ (see [2] for more details).

\section{STRIPPING FOIL HEATING}

Heating and cooling of an injection stripping foil due to irradiation with a pulsed beam is an essentially nonstationary phenomenon described with the following equation:

$$
\frac{d T}{d t}=\frac{N \delta_{b}\left(1-\delta_{e}\right)}{\rho c(T)}\left|\frac{d E}{d x}\right|-\frac{2 \epsilon \sigma_{\mathrm{SB}}}{\Delta x \rho c(T)}\left(T^{4}-T_{0}^{4}\right)
$$



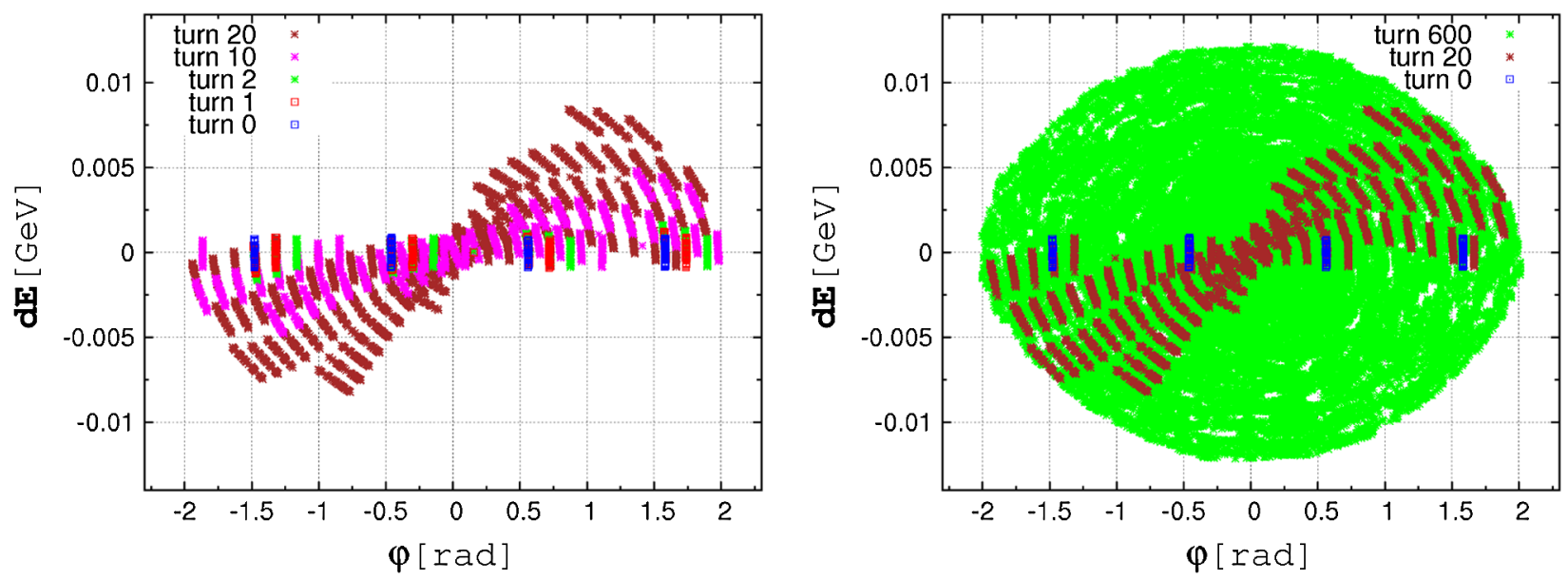

FIG. 7. Longitudinal particle distribution after $0,1,2,10$, and 20 turns (left) and after 0, 20, and 600 turns (right) for full beam current.

where $N$ is the beam hit density, $c(T), \rho$, and $\epsilon$ are the specific heat, density, and emissivity of carbon, respectively, $\left|\frac{d E}{d x}\right|$ is ionization energy loss of a beam proton, $\sigma_{\mathrm{SB}}$ is the StefanBoltzmann constant, $\Delta x$ is the foil thickness, $T$ and $T_{0}$ are the foil and ambient temperature, respectively, $\delta_{e}$ is the ratio of energy taken away by all secondary electrons (i.e. $\delta$ electrons) that escape the foil, to energy of all secondary electrons generated in it, and $\delta_{b}$ is 1 or 0 depending on whether the beam is on or off at the given time $t$. One assumes a constant hit rate within a single beam pulse. This equation is applied to the hottest spot on the foil and heat conductivity is ignored. Temperature dependence of carbon specific heat is taken from Ref. [7]. The consideration is limited to foil temperatures not exceeding $2500 \mathrm{~K}$ which means that foil failures due to evaporation are not taken into account [8].

For thin carbon foils-of order a few hundred $\mu \mathrm{g} / \mathrm{cm}^{2}$ - the number of secondary electrons that escape the foil is not negligible, which implies a tangible reduction in foil temperature. Therefore, special attention is paid to the generated secondary electrons and their transport, down to the lowest possible energy. The problem is in the energy distribution of the secondary electrons generated along the proton track, $d^{2} N / d E d x$, which is well known only for electron energies in the region $I \ll E \leq T_{\max }$ and behaves as $E^{-2}$, where $I$ is the mean excitation energy of the target atoms and $T_{\max }$ is maximum kinetic energy of a secondary electron according to kinematics. At very low energies, the distribution is barely known and can be approximately described as $E^{-\eta}$, with $\eta$ being approximately between 3 and 5 [9].

Thus, the value of $\delta_{e}$ is calculated in two different ways: (i) by Monte Carlo; (ii) analytically with some numerical (nonstochastic) integration. This gives us the possibility to study the sensitivity of the results to the electron energy cutoff and validate the Monte Carlo results.

If one considers the first passage of an injected $\mathrm{H}^{-}$ion through a stripping foil, the energy deposition by two stripped electrons is comparable to that by the proton. However, the same proton will make about a hundred more passages through the foil during the multiturn injection (see Table II), so that one can safely ignore the energy deposition by the stripped electrons.

\section{A. Calculation of absorbed energy by Monte Carlo}

The modeling of electron transport in the foil is performed with the MCNPX code [10] down to $1 \mathrm{keV}$ and with the MARS code $[11,12]$ down to $200 \mathrm{keV}$. In our model, $\delta_{e}$ is defined by the following expression:

$$
\delta_{e}=\frac{E_{\mathrm{out}}}{\frac{d E}{d x} \Delta x}
$$

where $E_{\text {out }}$ is energy taken away by all escaped electrons and defined by the following expression:

$$
E_{\text {out }}=\iiint E \Phi_{e}(\mathbf{r}, E, \Omega)|\hat{\mathbf{n}} \Omega| d A d E d \Omega
$$

where $\Phi_{e}(\mathbf{r}, E, \Omega)$ is the appropriately normalized electron flux density at the point $(\mathbf{r}, E, \Omega)$ of phase space, $\hat{\mathbf{n}}$ is a unit vector orthogonal to a surface of the foil, and $d A$ refers to integration over the foil surface. For protons with kinetic energy of a few $\mathrm{GeV}$, the ionization energy loss in thin foils is approximated by the simple product in the denominator of Eq. (2), that can be evaluated numerically using tables of ionization energy loss [13]. The value of $E_{\text {out }}$ is calculated in two different ways. For the MARS code, the calculation starts with protons incident on the foil and the $\delta$ electrons that escape the foil are counted. In the code MCNPX 2.6, secondary electrons are not generated explicitly by protons and other charged heavy particles. Therefore, in this case the calculation starts with the secondary electrons themselves and one assumes a uniform distribution of generated secondary electrons along the proton track as well as realistic dependence of angle vs energy according to kinematics of elastic proton-electron collisions [9]. Energy 


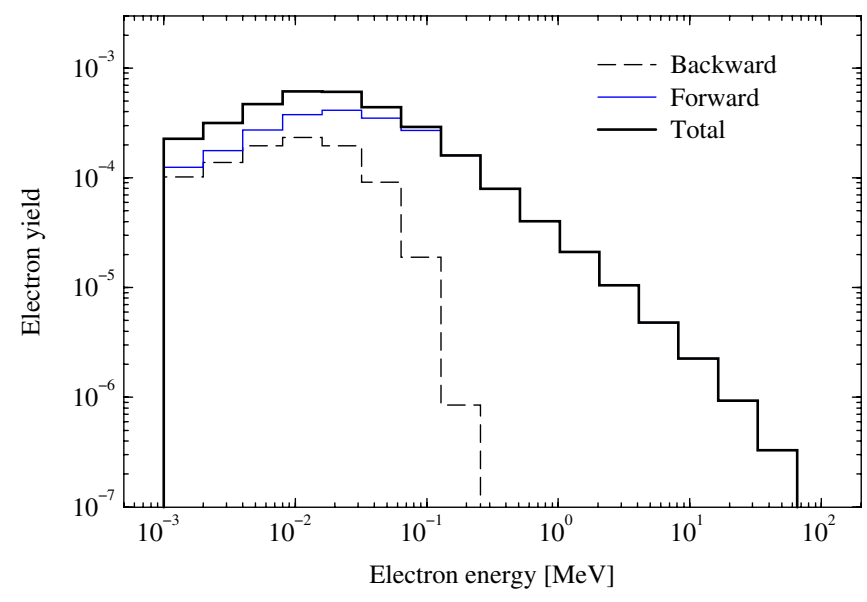

FIG. 8. Calculated energy distributions of secondary electrons that escape the carbon foil as thin as $600 \mu \mathrm{g} / \mathrm{cm}^{2}$ irradiated with an $8-\mathrm{GeV}$ proton beam at normal incidence [10]. Normalization is per incident proton.

distributions of electrons that escape the carbon foil are shown in Fig. 8. The calculated value of $\delta_{e}$ for $8-\mathrm{GeV}$ protons and a carbon foil as thin as $600 \mu \mathrm{g} / \mathrm{cm}^{2}$ is 0.281 (see also Tables III and IV).

\section{B. Nonstochastic calculation of absorbed energy}

A simple model [14], developed initially for low-energy $(50 \mathrm{MeV})$ protons, was modified for high energies in order to take into account relativistic effects. The differential cross section of $\delta$-electron production can be written as [9]

$$
\frac{d \sigma}{d E}=\frac{k}{E^{2}}\left(1-\beta^{2} \frac{E}{E_{\max }}+\frac{E^{2}}{2 E_{0}^{2}}\right),
$$

where $k=0.1535 Z /\left(A \beta^{2}\right) \mathrm{MeV} \mathrm{cm}^{2} / \mathrm{g}, A$ is target atomic mass, and one assumes that the speed of light in vacuum, $c$, is equal to unity. The maximum kinetic energy that an electron can receive in a collision, $E_{\max }$, is given by

$$
E_{\max }=2 m_{e} p_{0}^{2} /\left(m_{e}^{2}+M^{2}+2 m_{e} E_{0}\right)
$$

TABLE IV. The ratio of energy taken away by generated $\delta$ electrons that escape the carbon foil of the given thickness to total energy loss by a proton, $\delta_{e}$, according to model $M_{2}$ with cutoff of $0 \mathrm{keV}$. Ratio of energies deposited in the foil by generated $8 \mathrm{GeV}$ protons according to model $M_{2}$, calculated with cutoff energy of 200 and $0 \mathrm{keV}, E D_{200} / E D_{0}$.

\begin{tabular}{lcc}
\hline \hline Thickness $\left(\mathrm{g} / \mathrm{cm}^{2}\right)$ & $\delta_{e}$ & $E D_{200} / E D_{0}$ \\
\hline $10^{-4}$ & 0.313 & 1.14 \\
$4 \times 10^{-4}$ & 0.288 & 1.10 \\
$6 \times 10^{-4}$ & 0.281 & 1.09 \\
$8 \times 10^{-4}$ & 0.276 & 1.084 \\
$10^{-3}$ & 0.271 & 1.077 \\
$10^{-2}$ & 0.226 & 1.02 \\
$10^{-1}$ & 0.175 & 1.002 \\
1 & 0.109 & 1.0002 \\
\hline \hline
\end{tabular}

where $m_{e}$ is electron mass, $M, p_{0}$, and $E_{0}$ are proton mass, momentum, and total energy, respectively. Electrons are emitted at an angle of $\psi$ with respect to proton direction, that is given by

$$
\cos \psi=\sqrt{E /\left(E+2 m_{e}\right)}\left(E_{0}+m_{e}\right) / p_{0} .
$$

If an electron is produced at a point $z$ in a foil of thickness $t$, it will travel the distance $l=(t-z) / \cos \psi$ and escape from the foil with energy $W(E, l)$. Kobetich and Katz [15] proposed an empirical expression for energy deposition in the foil based on a fit to experimental data. According to their model, energy of $\delta$ electrons that escape the foil is

$$
E_{\text {out }}=\int_{E_{\mathrm{th}}}^{E_{\max }} d E \frac{d \sigma}{d E} \int_{0}^{t} d z W[E,(t-z) / \cos \psi]
$$

Note that Eq. (4) is inaccurate for kinetic energies close to mean excitation energy (approximately $70 \mathrm{eV}$ for carbon). However, such low-energy electrons are produced at nearly 90 degrees and cannot escape the foil because of their extremely low ranges. As shown in Table III, the

TABLE III. Energy $(\mathrm{keV})$ taken away by generated $\delta$ electrons that escape the carbon foil of a given thickness. Normalization is per incident $8-\mathrm{GeV}$ proton. The electron energy cutoff used in the calculations is shown in parentheses.

\begin{tabular}{lcccccr}
\hline \hline $\begin{array}{l}\text { Thickness } \\
\left(\mathrm{g} / \mathrm{cm}^{2}\right)\end{array}$ & $\begin{array}{c}\text { Model } M_{1} \\
(1 \mathrm{keV})\end{array}$ & $\begin{array}{c}\text { Model } M_{2} \\
(1 \mathrm{keV})\end{array}$ & $\begin{array}{c}\text { MCNPX } \\
(1 \mathrm{keV})\end{array}$ & $\begin{array}{c}\text { Model } M_{2} \\
(0 \mathrm{keV})\end{array}$ & $\begin{array}{c}\text { Model } M_{2} \\
(200 \mathrm{keV})\end{array}$ & $\begin{array}{c}\text { MARS } \\
(200 \mathrm{keV})\end{array}$ \\
\hline $10^{-4}$ & 0.0655 & 0.0579 & 0.0580 & 0.0578 & 0.0398 & 0.0385 \\
$4 \times 10^{-4}$ & 0.243 & 0.212 & 0.211 & 0.213 & 0.159 & 0.160 \\
$6 \times 10^{-4}$ & 0.357 & 0.311 & 0.308 & 0.310 & 0.238 & 0.239 \\
$8 \times 10^{-4}$ & 0.468 & 0.406 & 0.400 & 0.407 & 0.318 & 0.320 \\
$10^{-3}$ & 0.576 & 0.500 & 0.489 & 0.500 & 0.397 & 0.397 \\
$10^{-2}$ & 4.94 & 4.18 & 3.99 & 4.18 & 3.88 & 3.84 \\
$10^{-1}$ & 39.8 & 32.3 & 30.3 & 32.2 & 32.0 & 32.8 \\
1 & 274 & 202 & 189 & 202 & 204 & 208 \\
\hline \hline
\end{tabular}


TABLE V. Calculated hit density on a foil at the hottest spot for various injection cycles and for painting injection scenarios $A$ through $D$ (see Table II).

\begin{tabular}{lcccc}
\hline \hline Hit density, $10^{14}$ proton $/ \mathrm{mm}^{2}$ & $A$ & $B$ & $C$ & $D$ \\
\hline Cycle 1 & 0.09 & 0.17 & 0.35 & 5.15 \\
Cycle 2 & 0.17 & 0.33 & 0.66 & \\
Cycle 3 & 0.22 & 0.44 & 0.86 & \\
Cycle 4 & 0.26 & 0.51 & 1.02 & \\
Cycle 5 & 0.31 & 0.62 & 1.25 & \\
Cycle 6 & 0.34 & 0.68 & 1.34 & \\
All injection cycles & 1.31 & 2.58 & 5.14 & 5.15 \\
\hline \hline
\end{tabular}

results with threshold energies of 1 and $0 \mathrm{keV}$ practically coincide for carbon thicknesses more than $10^{-4} \mathrm{~g} / \mathrm{cm}^{2}$.

Calculated absolute values of the energy, taken away by $\delta$ electrons that escape the foil, are given in Table III. One studied two models, $M_{1}$ and $M_{2}$. The model $M_{1}$ means that, as in [14], only the first term in Eq. (4) was taken into account. This model overestimates the energy yield from the foil. If the first two terms in Eq. (4) are used $\left(M_{2}\right)$, the nonstochastic model and MCNPX simulations agree within a few percent for thicknesses from $10^{-4}$ to $1 \mathrm{~g} / \mathrm{cm}^{2}$. The contribution due to the third term in this equation is negligible.

The simulations performed with the MARS code agree well with the model $M_{2}$ for the threshold of $200 \mathrm{keV}$. Currently, it is the lowest electron cutoff energy in the MARS code. Table IV presents the ratio of escaped energy to total energy loss by a proton. It is seen that only $70 \%$ of proton ionization energy loss goes to target heating. High threshold calculations $(200 \mathrm{keV})$ overestimate the energy deposition by about $10 \%$ for absorbers as thin as $10^{-3} \mathrm{~g} / \mathrm{cm}^{2}$ and less, but provide good accuracy for thicknesses more than $10^{-2} \mathrm{~g} / \mathrm{cm}^{2}$.

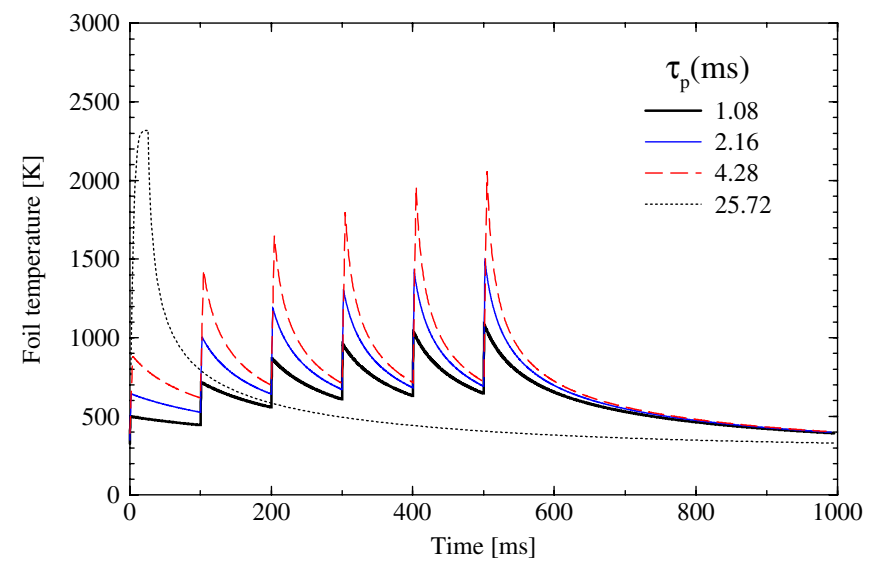

FIG. 9. Calculated temperature distributions during an injection cycle for several painting cycle durations, $\tau_{p}$ (see Table II). The temperatures are given at the hottest spot of the $600-\mu \mathrm{g} / \mathrm{cm}^{2}$ corner carbon foil irradiated with an $8-\mathrm{GeV}$ proton beam at normal incidence.
TABLE VI. Experimental data on fractional yields of excited states of $\mathrm{H}^{0}$ atoms due to foil stripping for incident $0.8-\mathrm{GeV}$ $\mathrm{H}^{-}$ions.

\begin{tabular}{lc}
\hline \hline Principal quantum number, $n$ & Yield, $\%$ \\
\hline 1,2 & 93.3 \\
3 & 3.6 \\
4 & 1.5 \\
5 & 0.7 \\
6 & 0.3 \\
$>6$ & 0.6 \\
Total & 100 \\
\hline \hline
\end{tabular}

\section{Thermal calculations}

Calculations of the beam hit densities, $N$, were performed with the STRUCT code for several painting scenarios (see Table V). In Table V the hit density given for all

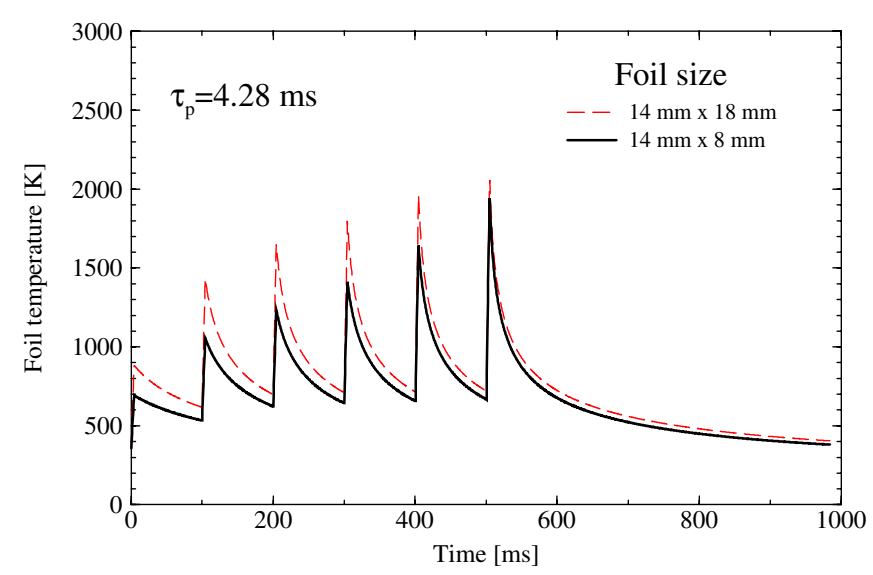

FIG. 10. Calculated temperature distributions for the $600-\mu \mathrm{g} / \mathrm{cm}^{2}$ corner carbon foils of different transverse sizes (painting scenario $C$ ) irradiated with an $8-\mathrm{GeV}$ proton beam at normal incidence.

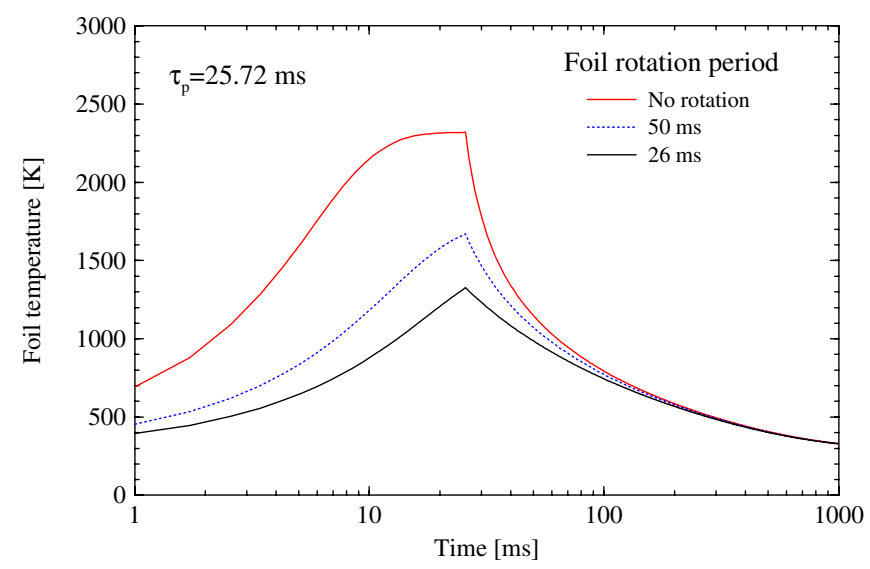

FIG. 11. Calculated temperature distributions for the $600-\mu \mathrm{g} / \mathrm{cm}^{2}$ corner stationary and circular rotating carbon foils (painting scenario $D$ ) irradiated with an $8-\mathrm{GeV}$ proton beam at normal incidence. 

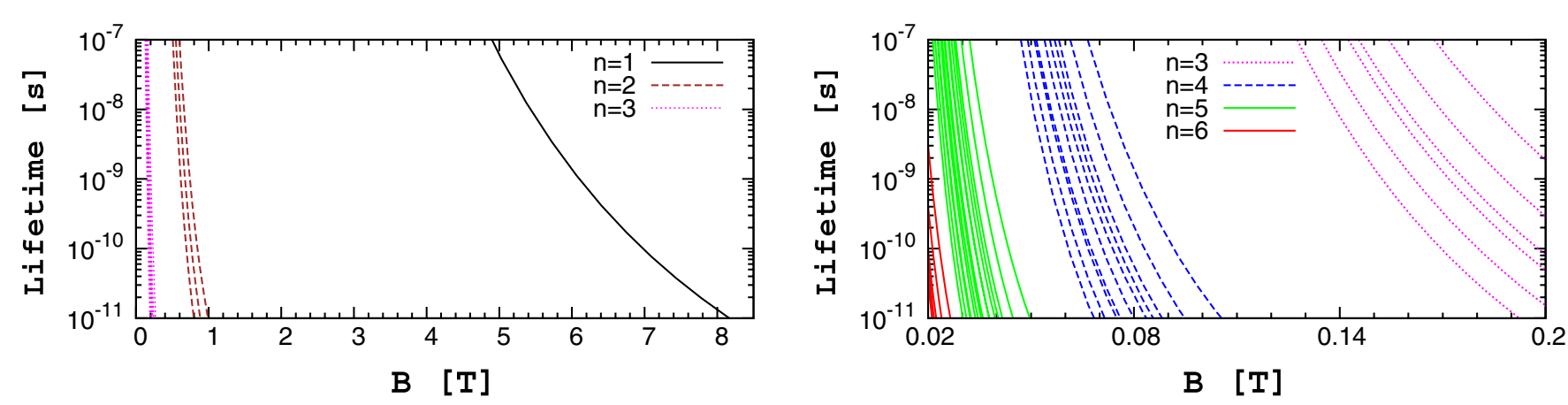

FIG. 12. Lifetime of various states of a neutral hydrogen atom in magnetic fields calculated in the laboratory frame for an atom with kinetic energy of $8 \mathrm{GeV}$.

injection cycles is for studying average foil heating and is not equal to sum of hit densities over all injection cycles, because location of the hottest spot can move around the foil during the injection painting.

Given the beam hit density, numerical integration of Eq. (1) is performed with the Runge-Kutta method [16]. Calculated temperature distributions at the hottest spot on the foil are shown in Fig. 9. Regarding the maximum temperature, for a stationary foil the painting injection scenarios $A$ and $B$ look the most viable.

One can see in Table IV that reduction of the foil thickness does not provide a substantial reduction in deposited energy. A reason for that is in the energy and angular distribution of the secondary electrons-a significant percentage of them is generated with low energies and at large angles relative to the incident proton direction (see also Fig. 8). A comparison of temperature profiles for two foils presented in Fig. 10 reveals that one can hardly achieve significant reduction in foil temperature by means of mechanical design optimization.

The painting scenario $D$ represents a relatively long injection at low intensity. In such a case, one could reduce the maximum temperature by means of foil rotation. A comparison between a stationary foil and rotating ones shown in Fig. 11 reveals that a tangible reduction in the maximum temperature can be achieved, so that the long low-intensity injection scenario can be of practical importance as well.

\section{EFFICIENCY OF FOIL STRIPPING}

After interaction with the foil, an unstripped outgoing part of the beam may be stripped to protons by the magnetic field of accelerator elements. The unstripped part consists of neutral hydrogen atoms both in ground and various excited states split due to the presence of the external field. Lifetimes of the excited atomic states in a magnetic field for $8 \mathrm{GeV}$ hydrogen atoms, calculated with Eq. (72) of Refs. [17,18], are shown in Fig. 12. The stripping foil is located at the exit of magnet $\mathrm{HBC} 2$ and the entrance of HBC3 (Fig. 2), very close to the edge in a fringe field of these magnets.
Detailed analysis of stripping probabilities for the excited atomic states in the HBC2 and $\mathrm{HBC} 3$ magnets is presented in Ref. [19]. We assume that an $\mathrm{H}^{0}$ atom travels a distance of at least $\sim 1 \mathrm{~cm}$ in the fringe field of these magnets. All atoms with $n \geq 2$, where $n$ is principal quantum number, are stripped to protons and go to the circulating beam without changing emittance of the beam because they are stripped along a very short path length downstream the foil. Atoms with $n=1$ are left unstripped. The probability for these atoms to be stripped is less than $10^{-10}$ and they go to the thick foil and to the beam absorber (see Fig. 2).

Experimental data on yields of various excited states of neutral hydrogen atoms, produced by foil stripping for incident $800 \mathrm{MeV} \mathrm{H}^{-}$ions [20], are presented in Table VI. The previously calculated stripping efficiency [21] is $99.5 \%$. Assuming that the fractional yields for different excited states $n$ do not depend on the foil thickness and energy, one may expect that about $90 \%$ - as conservatively estimated from Table VI-of the total yield of the excited atoms are those with $n=1$ which go to the beam absorber. Total power load in the absorber at normal operation is less than $0.5 \%$ of the injected beam power. The remaining $10 \%$ of the $\mathrm{H}^{0}$ atoms will be stripped to protons by magnetic fields of $\mathrm{HBC} 2$ and HBC3 magnets and will go into circulating beam without increasing emittance.

\section{BEAM LOSS IN THE TRANSVERSE DIRECTION}

The circulating protons pass several times through the foil and some of them can be lost because of multiple Coulomb scattering. Particle energy loss (ionization loss) in the foil at one pass is $\sim 4 \times 10^{-20}$ of initial energy. The rate of nuclear interactions in the foil during the total process is $\sim 8 \times 10^{-4}$ of injected intensity for 582-turn injection. The emittance of the circulating beam in the horizontal plane is small in the beginning of painting and it gradually reaches maximum only at the end of painting. But in a vertical plane the beam is close to the aperture during the total cycle of injection, because painting starts from large vertical amplitudes. Simulations show that the 
rate of particle loss in the accelerator due to interactions with the foil is as low as $\sim 3 \times 10^{-3}$ of the injected intensity for the 582-turn injection.

\section{CONCLUSIONS}

Multiturn $\mathrm{H}^{-}$stripping injection gives the only way to reach the beam intensities about $1.5 \times 10^{14}$ protons per pulse $(2.1 \mathrm{~A})$ in the recycler ring, which fills the main injector for further acceleration up to $120 \mathrm{GeV}$. The resulting beam power will bring the experimental program at Fermilab to the intensity frontier.

Several painting scenarios were studied numerically in terms of the duration and waveforms of the kicker magnets. The criteria were to minimize the number of hits and, correspondingly, the foil heating. For each scenario we performed a comprehensive analysis of the secondary electron flux and $\mathrm{H}^{0}$ production as well as did energy deposition and thermal calculations using both Monte Carlo and semianalytical approaches which agreed well. The cases of stationary and rotating foils were compared as well, and the rotation may help to reduce the temperature further and minimize beam losses.

For the time being, only the stripping foil is well established for such accelerator applications as multiturn injection. In the meantime, a laser stripping injection, which lacks many problems of a physical foil [22], may become an attractive option for future high intensity accelerator projects. However, the stripping of $\mathrm{H}^{-}$due to electron photo detachment requires at least a $10 \mathrm{MW}$ peak power laser with the stripping efficiency of about $98 \%$. So far, the foil stripping remains the principal option for injection in Project X.

\section{ACKNOWLEDGMENTS}

The authors wish to thank Jeffrey A. Holmes (SNS) for discussions on the ORBIT package, and critical comments from Peter Lucas (FNAL) are greatly appreciated. This work was supported by Fermi Research Alliance, LLC, under Contract No. DE-AC02-07CH11359 with the U.S. Department of Energy.

[1] http://projectx.fnal.gov/.

[2] A. I. Drozhdin, L. G. Vorobiev, and D.E. Johnson, in Proceedings of the 23rd Particle Accelerator Conference, Vancouver, Canada, 2009 (IEEE, Piscataway, NJ, 2009), pp. 1418-1420.

[3] I. Baishev, A. Drozhdin, N. Mokhov, and X. Yang, Report No. MAN-0034, SSCL, 1994 [http://www-ap.fnal.gov/ users/drozhdin/].
[4] Y. Yamazaki, Y. Mori, Y. Irie, T. Kato, and S. Noguchi, Technical Report No. 97-16, KEK, 1998.

[5] J.D. Galambos, J.A. Holmes, D.K. Olsen, A. Luccio, and J. Beebe-Wang, Technical Report, SNS/ORNL, 1999.

[6] The MAD Program (Methodical Accelerator Design), Version 8.21, CERN (2002) [https://mad.web.cern.ch/mad/].

[7] Y.S. Touloukian and E.H. Buyco, Specific Heat. Nonmetallic Solids (IFI Plenum, NY-Washington, 1970).

[8] S. G. Lebedev and A. S. Lebedev, Phys. Rev. ST Accel. Beams 11, 020401 (2008).

[9] H. Bichsel, D. E. Groom, and S. R. Klein, Phys. Rev. D 66, 010001 (2002).

[10] Monte Carlo All-Particle Transport Code System MCNPX 2.6.0 (CCC-746), Los Alamos National Laboratory, Los Alamos, New Mexico, USA (2008).

[11] N. V. Mokhov, The MARS code system useŕs guide version 13(95), Fermilab-FN-628, 1995 [http://www-ap.fnal.gov/ MARS/].

[12] N. V. Mokhov and S. I. Striganov, in Hadronic Shower Simulation Workshop Proceedings, Batavia, Illinois, 2006 (American Institute of Physics, Melville, New York, 2007), pp. 50-60, Vol. 896.

[13] http://www.nist.gov/pml/data/index.cfm/.

[14] N. Laulainen and H. Bichsel, Nucl. Instrum. Methods 104, 531 (1972).

[15] E. J. Kobetich and R. Katz, Nucl. Instrum. Methods 71, 226 (1969).

[16] W.H. Press, S. A. Teukolsky, W.T. Vetterling, and B.P. Flannery, Numerical Recipes in C++. The Art of Scientific Computing (Cambridge University Press, Cambridge, 2002), 2nd ed.

[17] R.J. Damburg and V. V. Kolosov, in Rydberg States of Atoms and Molecules, edited by R. F. Stebbings and F. B. Dunning (Cambridge University Press, Cambridge, 1983), pp. 31-71.

[18] W. Chou and A. I. Drozhdin, Fermilab Beams Document 2202 (2008).

[19] A. Drozhdin, I. Rakhno, S. Striganov, and L. Vorobiev, Technical Report No. Fermilab-FN-0899-APC, Fermilab, 2011.

[20] M. S. Gulley, P.B. Keating, H.C. Bryant, E.P. MacKerrow, W. A. Miller, D. C. Rislove, S. Cohen, J. B. Donahue, D. H. Fitzgerald, S. C. Frankle, D. J. Funk, R. L. Hutson, R. J. Macek, M. A. Plum, N. G. Stanciu, O. B. van Dyck, C. A. Wilkinson, and C. W. Planner, Phys. Rev. A 53, 3201 (1996).

[21] W. Chou and H. Bryant, Technical Report No. Conf-05225-AD, Fermilab, 2005; Proceedings of the 21 st Particle Accelerator Conference, Knoxville, 2005 (IEEE, Piscataway, NJ, 2005), pp. 1222-1224.

[22] D.E. Johnson, A. I. Drozhdin, I. Rakhno, L. G. Vorobiev, T. Gorlov, and D. Raparia, in Proceedings of High-Intensity and High-Brightness Hadron Beam Workshop (HB 2010), Morschach, Switzerland (2010), pp. 162-166 [http://hb2010.web.psi.ch/proceedings/index .htm]. 